\title{
Setting the target for pemphigus vulgaris therapy
}

\author{
Christoph T. Ellebrecht and Aimee S. Payne \\ Department of Dermatology, University of Pennsylvania, Philadelphia, Pennsylvania, USA.
}

Despite the rising incidence of autoimmunity, therapeutic options for patients with autoimmune disease still rely on decades-old immunosuppressive strategies that risk severe and potentially fatal complications. Thus, novel therapeutic approaches for autoimmune diseases are greatly needed in order to minimize treatment-related toxicity. Such strategies would ideally target only the autoreactive immune components to preserve beneficial immunity. Here, we review how several decades of basic, translational, and clinical research on the immunology of pemphigus vulgaris (PV), an autoantibody-mediated skin disease, have enabled the development of targeted immunotherapeutic strategies. We discuss research to elucidate the pathophysiology of PV and how the knowledge afforded by these studies has led to the preclinical and clinical testing of targeted approaches to neutralize autoantibodies, to induce antigen-specific tolerance, and to specifically eliminate autoreactive B cells in PV.

\section{Pemphigus as a model autoantibody-mediated disease}

Autoimmunity is an incurable condition affecting $5 \%-8 \%$ of the Western population (1). For decades, the standard of care has relied on chronic immunosuppression, which causes significant morbidity and mortality (2-4). The ideal therapy would eliminate only disease-causing autoimmune cells while sparing immune cells that provide protective immunity, a strategy that is commonly referred to as "targeted therapy." Pemphigus vulgaris (PV) is among the best-characterized human autoimmune diseases with regard to immune repertoire profiling and the well-defined nature of the disease autoantigen, which makes it an ideal disease to develop targeted therapeutic approaches.

$\mathrm{PV}$ is a potentially fatal antibody-mediated autoimmune disease that is characterized by loss of cell adhesion (also known as acantholysis) in skin and mucous membranes (Figure 1) (5). Diagnosis is readily made by histology, which shows a pathognomonic suprabasal epithelial blister characterized by a "row of tombstones," representing keratinocytes that have lost intercellular contact due to disruption of desmosome adhesion but remain attached to the basement membrane zone by means of their hemidesmosomes. Autoantibodies in PV target the desmosomal cadherin desmoglein 3 (DSG3) in stratified epithelia, as well as DSG1 in mucocutaneous disease (6), and their binding to the keratinocyte cell surface can be clinically documented by direct immunofluorescence studies on patient skin samples or by indirect immunofluorescence studies using patient serum on various epithelial substrates. The presence of serum DSG3 autoantibodies identifies PV patients with a specificity and sensitivity of $98 \%-100 \%$ ( 7 ,

Conflict of interest: A.S. Payne and C.T. Ellebrecht are inventors on patents related to targeted therapy of autoimmune diseases (PCT/ US15/28872), including pemphigus. A.S. Payne has received research funding from Sanofi for the development of cellular immunotherapies for pemphigus.

Published: March 9, 2017

Reference information: JCI Insight. 2017;2(5):e92021. https:// doi.org/10.1172/jci.insight.92021 8); thus, nearly all PV patients but no unaffected individuals demonstrate detectable DSG3 reactivity.

Although autoreactivity to various other autoantigens in pemphigus has been described previously (9), DSG3 autoantibodies represent the major etiologic culprit of the disease, as several lines of evidence have shown they are both necessary and sufficient for the induction of acantholysis. Passive transfer of PV IgG into mice causes suprabasal skin blistering similar to that in human disease (10), which can also be induced by transfer of affinity-purified DSG3-specific antibodies and is abrogated after depletion of these antibodies from PV sera $(11,12)$. Autoantibodies against other autoantigens may also synergize with anti-DSG3 antibodies to cause epidermal damage (13). Collectively, these data establish PV as a model autoantibody-mediated disease, given that the disease autoantigen in PV is well defined, the pathogenic role of anti-DSG3 antibodies in PV has been clearly established, and the diagnosis of the disease is straightforward, with commercially available histology, immunofluorescence, and ELISA studies. 


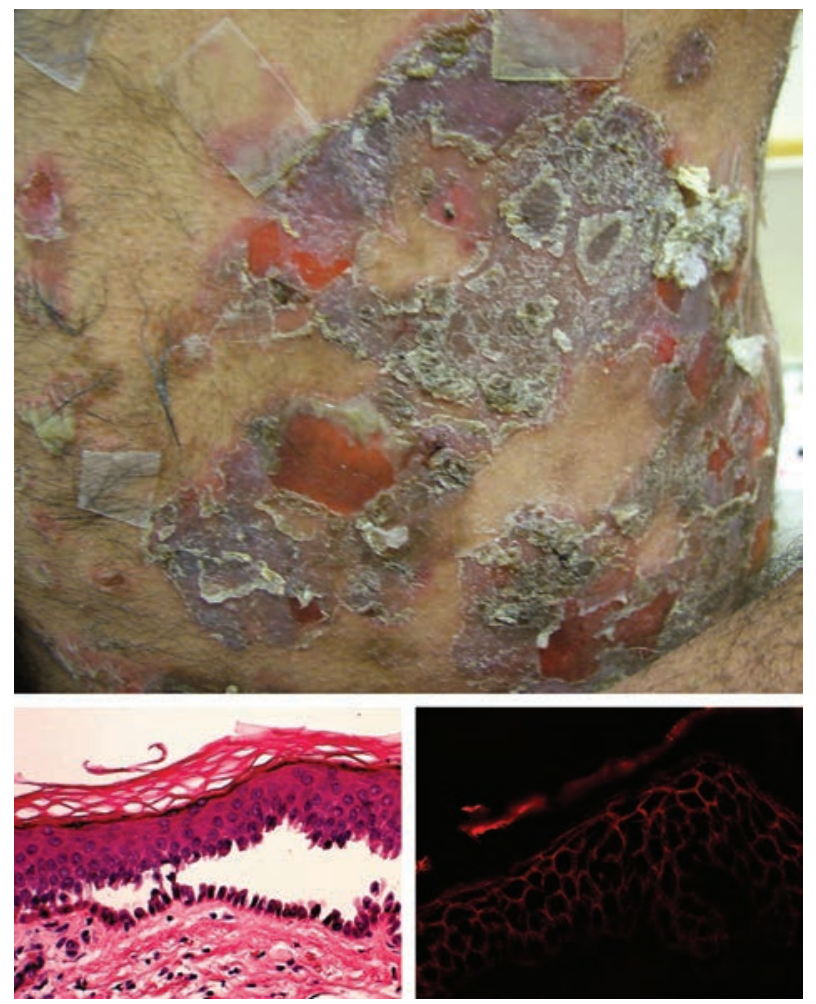

\section{Pathophysiologic mechanisms in PV}

As opposed to other autoantibody-mediated skin diseases, such as bullous pemphigoid or epidermolysis bullosa acquisita, monovalent autoantibody fragments are sufficient to induce acantholysis in animal and human skin models, indicating that neither Fc $\gamma$ receptor engagement nor complement activation is required for blister formation $(14,15)$. This observation is further underscored by the ability of autoantibodies to cause blisters in complement-deficient mice (16) as well as the predominance of IgG4 among PV autoantibodies (17, 18), a subclass that does not activate complement (19) and poorly binds to Fc receptors (20). Autoantibody binding to DSG3 causes skin blisters through several mechanisms, including direct interference with desmosomal adhesion by binding residues involved in trans- and cis-adhesive interactions (21-23), prevention of desmosome assembly or promotion of disassembly through clustering and/or endocytosis of DSG3 (24-27), and secondary activation of signal transduction events in keratinocytes, which augments the blistering response (28-31).

Because the autoantibody variable regions are sufficient for pathogenicity, much effort has been devoted to cloning immune repertoires from patients and PV model mice in order to characterize the autoreactive B cell populations (15, 21, 23, 32-34). All patient-derived anti-DSG3 mAbs whose epitopes have been reported bind to the amino-terminal extracellular cadherin (EC) domains, most often EC1 and EC2, where residues important for trans- and cis-adhesion reside $(35,36)$. The majority of B cell clones identified to date show patterns of somatic mutation consistent with an antigen-driven process $(15,23,33)$, although some B cell clones, particularly those using the antibody heavy chain gene VH1-46, demonstrate few somatic mutations and some do not require those mutations to bind to DSG3 (34). The genetic and functional characterizations of B cell repertoires in PV have provided not only a deeper mechanistic understanding of PV pathogenesis, but also valuable in vivo data on the diversity of human anti-DSG B cells, which have facilitated the development of targeted therapeutic approaches in PV.

Regarding the autoreactive $\mathrm{T}$ cell repertoire in PV, DSG3-specific Th2-type CD4 ${ }^{+} \mathrm{T}$ cells that recognize immunodominant DSG3 peptides have been identified in PV patients $(37,38)$. The strongest genetic determinant of PV susceptibility is in the HLA locus (39), most notably HLA-DRB1*04:02 in Jewish individuals (40), DQB1*05:03 in non-Jewish individuals of mixed European descent $(41,42)$, and DRB1*14 and DQB $1 * 05: 03$ in Japanese individuals $(43,44)$. HLA-DRB1*04:02 has been shown to bind an immunodominant DSG3 peptide (amino acids 190-204) in a manner particularly suitable for T cell activation (45). Interestingly, DSG3-specific Th1 cells can not only be detected in PV patients, but also in healthy individuals (38), whereas IL-10-producing DSG3-specific T cells with a regulatory phenotype are decreased in PV patients compared with healthy controls (46). DSG3 is expressed in the thymus under the AIRE promoter (47), which normally should delete DSG3-reactive T cells during central tolerization. These data suggest that peripheral tolerance can compensate for leaky central tolerance mechanisms to protect against DSG3 autoreactivity.

\section{Current PV treatment and implications for the development of targeted therapies}

In the presteroid era, the vast majority of PV patients died from their disease (48). A more recent population-based study indicates the 1 -year mortality for pemphigus is $12 \%$, representing a 3.4 hazard ratio for death in PV patients compared with age- and sex-matched controls (4). Current treatment strategies include topical and systemic corticosteroids in combination with other immunosuppressants, 


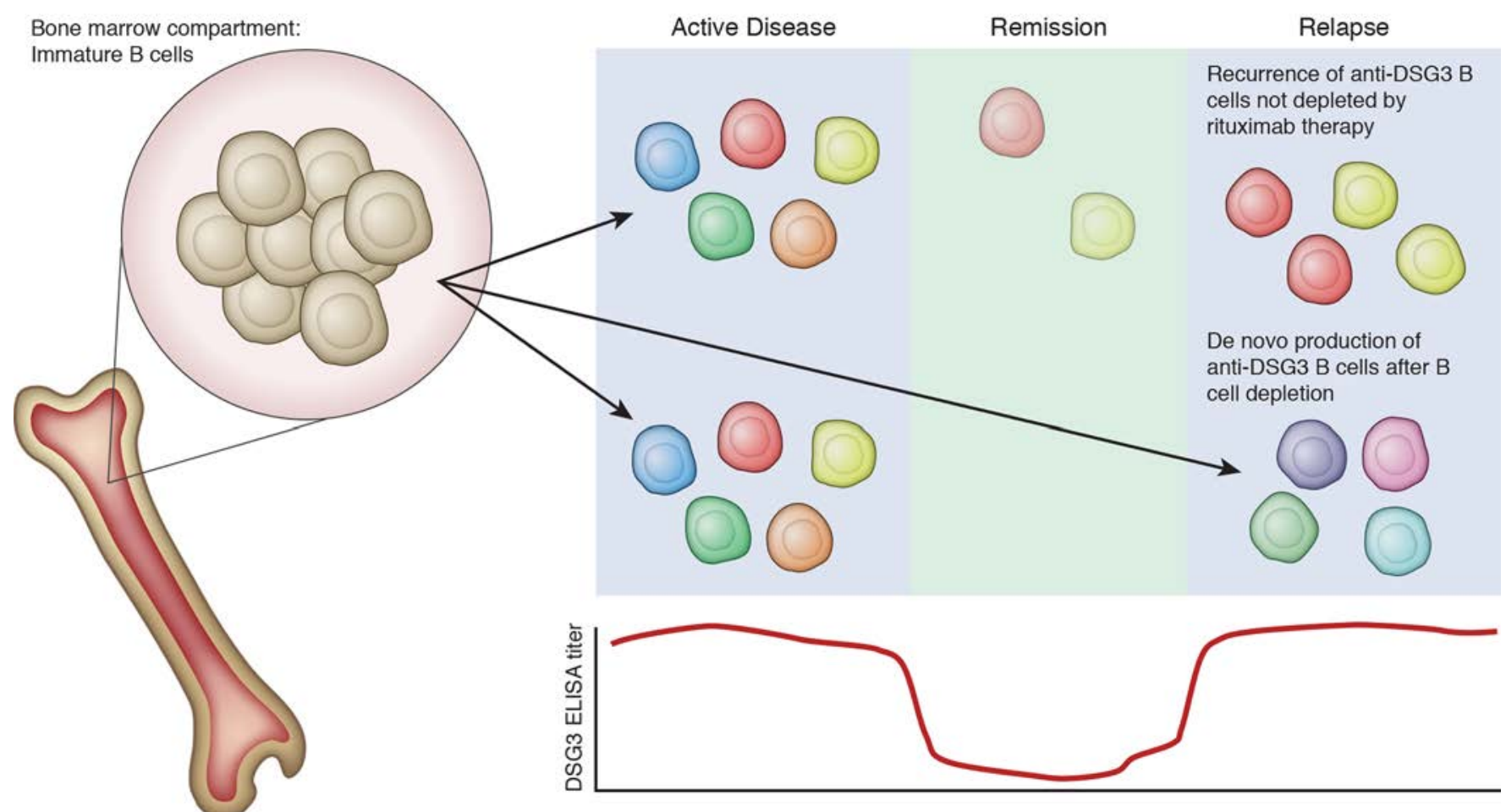

Figure 2. Potential mechanisms of disease relapse after $B$ cell depletion therapy. Relapse after rituximab therapy of pemphigus vulgaris may be due to incomplete B cell depletion and recurrence of the same anti-DSC3 B cells observed during initial disease, a model best supported by the current data. Alternatively, disease relapse may be triggered by the appearance of a new anti-DSG3 B cell repertoire after complete B cell depletion. Production of antiDSC3 antibodies by long-lived plasma cells, which are CD20- and hence not targeted by rituximab, appears not to play a significant role for many patients, given the serologic remissions of disease observed after rituximab therapy. Illustrated by Rachel Davidowitz.

most often azathioprine or mycophenolate, although only a few randomized clinical trials have proven their efficacy $(49,50)$. Treatment can be augmented with intravenous immunoglobulin $(51)$ or plasmapheresis (52-54), which aims to reduce circulating autoantibody levels. Treatment with the anti-CD20 antibody rituximab has been shown to be effective in inducing short-term disease remission in $95 \%$ $100 \%$ of PV patients $(55,56)$ associated with a decrease in anti-DSG antibody titers, while antibody titers to tetanus toxoid or Pneumococcal polysaccharides remain constant (57-59). This is a particularly interesting finding, as it indicates that DSG3 autoantibodies are produced by short-lived plasma cells that are continuously replenished from the $\mathrm{CD} 20^{+}$memory B cell pool. However, $81 \%$ of pemphigus patients experience disease relapse after rituximab (55), and analyses of rituximab use in pemphigus and other autoimmune diseases demonstrated a $1.3 \%-1.9 \%$ rate of fatal infection $(60,61)$, indicating the need for safer and more durable therapies.

Disease relapse after rituximab therapy can be explained by incomplete B cell depletion in bone marrow and secondary lymphoid organs (a known limitation of rituximab efficacy; ref. 62), by the emergence of newly formed DSG3-autoreactive B cell clones during B cell repopulation after complete $\mathrm{B}$ cell depletion, or by the production of autoantibodies by long-lived plasma cells that are CD20 negative and hence unaffected by CD20-targeted B cell depletion. If disease relapse is caused by the persistence and reexpansion of autoreactive B cells that are not effectively eliminated by rituximab, then strategies to completely eliminate DSG3-reactive B cells are likely to induce long-term remission in PV patients, since new anti-DSG3 B cells would typically not form. Conversely, if PV patients have an ongoing defect in tolerance to DSG3, new anti-DSG3 B cell clones would be expected to develop during each relapse after rituximab therapy. In this more challenging scenario, perpetual therapy would be required to control the development of autoreactive B cells (diagrammed in Figure 2). Additionally, if long-lived plasma cells contribute to anti-DSG antibody formation, then serologic remissions after rituximab therapy would not be observed, as this population would survive rituximab therapy, causing persistent blistering and elevations of serum autoantibody titers. 
A DSG3-specific adsorber

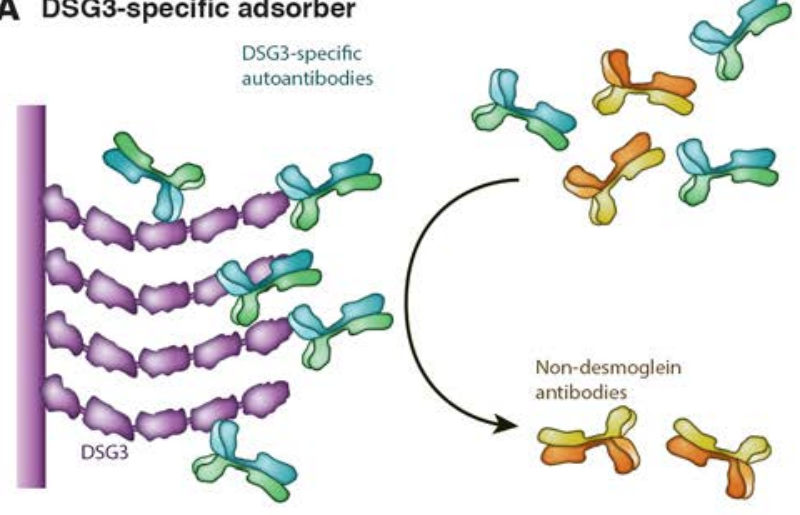

B DSG3-conjugated to Pseudomonas toxin A

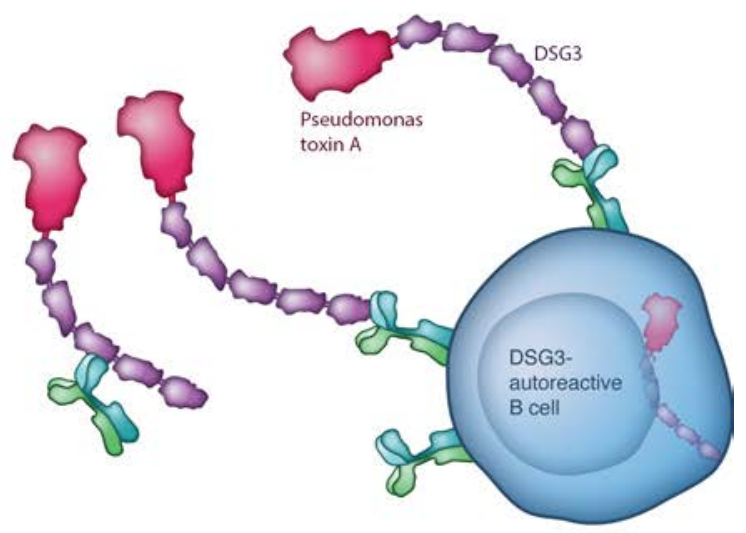

C DSG3 Chimeric Autoantibody Receptor T cell

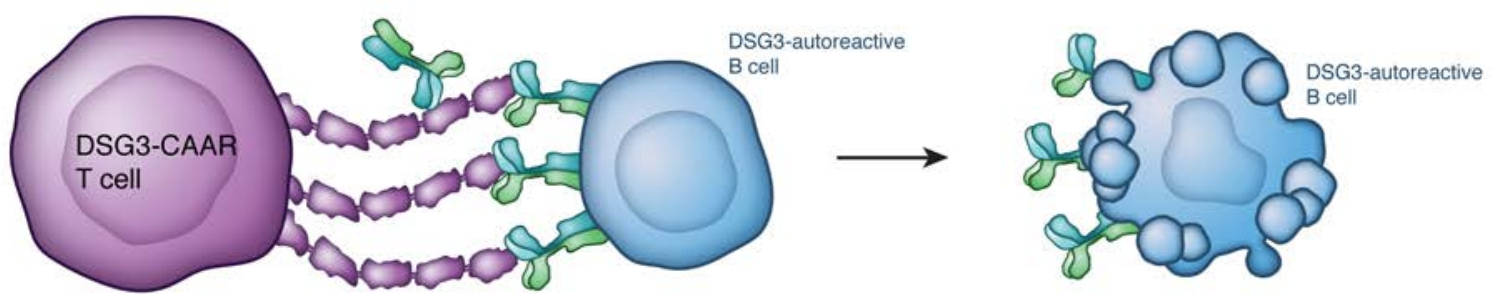

Figure 3. Autoantigen-based targeted therapies in pemphigus. Select strategies are illustrated that employ the autoantigen as a method for specific depletion of serum autoantibodies by immunoadsorption (A), targeted cell death of anti-DSC3 B cells by infusion of toxin-conjugated autoantigens that bind to anti-DSC3 B cell receptors (B), or durable elimination of anti-DSG3 B cells using DSC3-based cellular immunotherapy, also known as chimeric autoantibody receptor (CAAR) T cell therapy (C). Illustrated by Rachel Davidowitz.

Several lines of evidence suggest that the first scenario, in which there is a finite cohort of autoreactive B cells that persists over time to cause disease relapse, applies in PV. Longitudinal epitope-mapping studies of PV sera have shown that the targeted epitopes in DSG3 stay the same during active disease and relapse (63). Epitope spreading therefore appears to be a rare event, underscoring the static nature of the DSG3-reactive B cell repertoire in PV. Furthermore, cloning of DSG3-reactive B cell repertoires over time demonstrated that the same anti-DSG3 B cell clones are found during active disease and disease relapse, indicating that new autoreactive B cells generally do not appear over time and anti-DSG3 B cells are no longer detectable in the peripheral blood once patients achieve long-term remission (64). Finally, despite disease relapse in most rituximab-treated patients, PV patients can achieve both clinical and serologic remission after repeat courses of rituximab, indicating that $\mathrm{CD} 20^{-}$long-lived plasma cells do not appear to significantly contribute to autoantibody production, perhaps more so in patients treated early in the course of their disease $(55,59)$.

Taken together, the basic, translational, and clinical research studies in the field have considerably expanded our understanding of PV pathogenesis and positioned PV to be a model autoimmune disease for the development of targeted therapeutic strategies that can avoid systemic immunosuppression. Below, we discuss strategies to therapeutically target various elements of the PV autoimmune response without affecting the immune system as a whole, which would ideally prevent the severe side effects and deaths associated with current PV therapy.

\section{Autoantibody targeting strategies}

Because the antigenic specificity of the autoantibodies in PV is so well defined, strategies to either counteract or deplete these autoantibodies have been explored. Based on the knowledge that the amino-terminal epitopes of DSG3 are predominantly targeted by PV autoantibodies $(11,63)$, a tandem cyclic peptide consisting of DSG1 residues 81-87, highly homologous to the comparable DSG3 adhesive domain, was synthesized and shown to stabilize the trans-adhesive interface of both DSG3 and DSG1, thereby preventing autoantibody-mediated disruption of desmosomes $(30,65)$. This treatment was effective in preventing keratinocyte pathology in vitro and also epidermal blistering when applied topically in vivo. Additionally, 
the application of the tandem peptide prevented downstream signaling events that are usually observed after autoantibody binding. In a clinical setting, this strategy would serve as an adjunctive therapy to bolster keratinocyte adhesion in response to PV autoantibodies, but disease remission would still depend on standard immunosuppressive therapy to reduce the circulating autoantibodies. A potential drawback of such a strategy would be the possible immunogenicity of the tandem peptide, which could result in antibodymediated neutralization. Additionally, since the tandem peptide was designed to mimic the DSG transadhesive interface, newly formed antibodies could theoretically exacerbate acantholysis by stimulating the autoimmune response against a critical DSG3 functional domain.

As an alternative approach to autoantibody-targeted therapy, the finding that affinity-mediated adsorption of DSG3 autoantibodies from PV sera abrogates disease induction (12) led to the development of immunoadsorption columns conjugated to DSG3 or DSG1 and truncated variants spanning EC1 and EC2 to extract PV autoantibodies from patients' plasma (Figure 3A) (66). Despite concentration of targeted epitopes in EC1-2, the best depletion of plasma antibodies was observed when full-length DSG3 and DSG1 were used for immunoadsorption, suggesting that conformational epitopes recognized by PV autoantibodies (67) were not sufficiently reproduced when using their truncated DSG fragments. However, a construct consisting of only DSG1 EC1 and EC5 was recognized by 12 of 13 pemphigus foliaceus (PF) patient sera, consistent with the observation that an EC1-targeted $\mathrm{mAb}$ has been shown to inhibit binding of the majority of polyclonal PF sera (68).

Using antigen-specific immunoadsorbers that exclusively extract the disease-causing autoantibodies represents an attractive approach to therapy, as it avoids hypogammaglobulinemia associated with non-specific immunoadsorption strategies and hence the risks of generalized immunosuppression. An additional advantage of antigen-specific immunoadsorption is the reduction in complications due to fibrinogen fluctuation after the procedure compared with plasma exchange (69). An unanswered question in the context of antigen-specific immunoadsorption is whether release of the autoantigen from the adsorber into the serum could modify the immune response to DSG3, either through a tolerizing and therefore beneficial effect or a deleterious exacerbation of the immune response against DSG3. Both outcomes appear possible, as studies have shown that autoantigen presentation without costimulatory signals results in tolerance induction; however, tolerance-inducing strategies using peptide or otherwise modified autoantigens can induce disease flares in other autoimmune conditions (70).

Another approach that has been explored to target the soluble DSG3-specific autoantibodies in PV utilizes anti-idiotype antibodies (71) raised in rabbits by immunization with monoclonal anti-DSG single-chain variable fragments cloned from PV patients. As shown by ELISA and keratinocyte dissociation assays, pooled anti-idiotype antisera inhibited antibody binding to DSG3 and acantholysis induced by the serum IgG of the patient from whom the antibodies were cloned but not serum IgG from two other PV patients. Nevertheless, pathogenic serum IgG was eluted from the antisera-conjugated immunoadsorption columns from all $3 \mathrm{PV}$ patients, suggesting that this approach identified some but not all of the pathogenic antibodies in other PV patients (71). These data indicate that the B cell idiotypes in PV are likely too diverse to be therapeutically targeted in all patients either through a specific anti-idiotype or through more general VH gene-targeted reagents, although personalized approaches to identify the scope of B cell idiotypes within each patient could be effective. These reagents could be utilized in several therapeutic applications: as a method of anti-DSG antibody adsorption, as soluble mAbs that would work similarly to rituximab and provide the advantage of only depleting anti-DSG B cell populations through their surface B cell receptor (BCR), or as chimeric immunoreceptors that direct $\mathrm{T}$ cell cytolysis against specific $\mathrm{B}$ cell populations (discussed further below). A drawback of using such reagents as soluble infused antibodies is the formation of immune complexes between DSG3-specific and anti-idiotype antibodies, which could exacerbate the autoimmune response or cause other immune complex-mediated complications (72).

\section{Antigen-specific tolerance induction}

As antigen-specific autoantibody production is thought to result from $\mathrm{T}$ cell stimulation of B cells, efforts to induce antigen-specific tolerance hold promise as methods to interfere with all aspects of the detrimental immune response. However, there are challenges facing this approach, particularly in autoantibody-mediated diseases in which immune complex formation between soluble autoantibodies and administered autoantigen could result in immune complex-mediated exacerbation of disease. Efforts to induce tolerance in various autoimmune diseases by administration of autoantigen have shown variable efficacy and some flares 
of disease (70). One notable success is in multiple sclerosis (MS), which has led to a phase I clinical trial in humans using peripheral blood mononuclear cells (PBMCs) chemically engineered to present peptides derived from presumptive MS autoantigens in the absence of costimulatory signals (73). The trial demonstrated the safety and tolerability of this strategy, and the patients showed a lower frequency of antigenspecific $\mathrm{T}$ cells after treatment with increasing doses of tolerizing PBMCs, suggesting that pathogenic T cells had been deleted.

In $\mathrm{PV}$, one human clinical trial has explored the safety and feasibility of a peptide vaccine strategy to induce DSG3-specific immune tolerance (74). A peptide encompassing amino acids 186-204 of DSG3, predicted to specifically bind to the pocket of HLA-DRB1*0402 (45), was evaluated in a phase I trial in PV patients carrying the DRB1*0402 allele to determine if it would induce tolerance in $\mathrm{CD}^{+} \mathrm{T}$ cells through the MHCII/T cell receptor interaction in the absence of costimulatory signals. The treatment was generally safe and well-tolerated. Two of seventeen patients showed disease flares during a 5-month follow-up period; however, no significant change in autoantibody titers against DSG3 was observed, and no further clinical development of this strategy has been pursued.

Although not tested in PV, it is worthwhile to discuss the clinical success of allergen-specific tolerance induction by systemic desensitization (75) and whether similar approaches could work in PV. Exposure to increasing doses of allergen has been shown to divert the B cell response from anaphylaxis-causing IgE to blocking IgG4 antibodies that lack effector function $(76,77)$. However, as discussed above regarding the pathophysiology of pemphigus, such a strategy may not work in PV since acantholysis induced by PV autoantibodies does not depend on the antibody Fc. Moreover, since the majority of PV autoantibodies already belong to the IgG4 subclass, a deviation of the immune response by chronic antigen stimulation to IgG4 is not likely to ameliorate and may worsen the course of PV.

An intriguing strategy for antigen-specific tolerization may be the utilization of autoantigen-specific Tregs that suppress DSG3-specific immune responses, an approach supported by the finding of decreased DSG3-specific Tregs in PV patients relative to unaffected individuals (46). Tregs that suppress HLA-specific $\mathrm{T}$ cell responses by means of an anti-HLA chimeric antigen receptor (CAR) have shown promise in preclinical studies (78), although strategies to avoid broader-than-intended immunosuppression must be considered.

\section{Antigen-specific B cell depletion}

Since anti-DSG B cells produce the disease-causing antibodies in PV, targeting antigen-specific B cells represents an extremely attractive approach that is likely to be effective, given the short-term efficacy of CD20-mediated B cell depletion in PV, and much safer than other B cell depletion strategies due to the preservation of non-DSG-reactive B cells by this approach. As discussed previously, short-lived plasma cells are the key antibody-secreting cells in PV and are dependent on the CD20 $\mathrm{B}$ cell pool for replenishment. Mature $\mathrm{CD} 20^{+} \mathrm{B}$ cells express BCRs on their surface, consisting of the same IgG molecules that the $\mathrm{B}$ cell will secrete once activated to mature into a plasma cell, except membrane anchored by means of a transmembrane domain. BCRs mediate the activation of the B cell upon antigen encounter, and this activation is associated with internalization of the BCR together with the bound antigen followed by processing of the antigen and presentation of the antigen-derived peptides in the context of $\mathrm{MHCII}$ to $\mathrm{CD} 4^{+} \mathrm{T}$ helper cells (79). PV-specific B cells will express a DSG3-specific BCR on their surface, which labels them unequivocally as autoreactive in PV, and can be exploited for antigen-specific B cell targeting. As discussed previously, prior clinical and translational studies on rituximab efficacy in pemphigus predict that such an approach should effectively eliminate the critical autoreactive B cell populations in PV, resulting in both clinical and serologic disease remission.

An initial study targeting the BCRs of DSG3-specific B cells in PV was performed using chimeric proteins consisting of the DSG3 extracellular domains fused to Pseudomonas exotoxin A (PE) (80). PE mediates its toxic effect by ADP-ribosylation of elongation factor 2 (EF2), which inhibits ribosomal protein synthesis in the host cell $(81,82)$. Binding of DSG3-PE to anti-DSG3 BCRs triggers internalization and processing of $\mathrm{PE}$, resulting in inhibition of protein translation in anti-DSG3 B cells and thus targeted cell death (Figure 3B). Incubation of DSG3-PE with mouse hybridomas that express surface anti-DSG3 BCRs, as well as B cells derived from DSG3-immunized mice, resulted in a $40 \%-60 \%$ reduction in antiDSG3 B cells after 48 hours. Killing was enhanced by inclusion of a carboxy terminal KDEL sequence that facilitates trafficking of proteins to the endoplasmic reticulum (83). The incomplete killing of anti-DSG3 B cells by DSG3-PE was postulated to be due to incomplete processing of the toxin and antibody-mediated 
neutralization of the soluble chimeric construct, the latter of which presents a major in vivo challenge to the therapeutic approach, as the soluble antibodies have to be overcome to induce a therapeutic effect at the $\mathrm{B}$ cell level and to avoid risks of immune complex formation. Additionally, the administration of a foreign protein such as PE can induce potent antibody responses (84), further limiting the efficacy of chimeric soluble proteins for targeted therapy.

We recently developed an approach to use the DSG3 autoantigen to target autoreactive B cells in PV by incorporating DSG3 into a chimeric immunoreceptor that can direct a patient's own T cells to specifically kill autoantigen-specific B cells (Figure 3C), a strategy known as chimeric autoantibody receptor (CAAR) technology (85). CAARs were developed by re-engineering CARs, which had previously shown success for cancer therapy. CARs consist of an antigen-specific antibody fragment on the surface of the $\mathrm{T}$ cell fused to a cytoplasmic $\mathrm{CD} 3 \zeta$ signaling domain, which directs $\mathrm{MHC}$-independent $\mathrm{T}$ cell cytolysis against cells expressing the antigen of interest (86) and whose efficacy can be potentiated through incorporation of costimulatory signaling domains derived from CD28 or CD137 $(87,88)$. Such approaches using anti-CD19 CAR-T cells against B cell cancers have shown remarkable clinical efficacy, with reports of long-lasting remissions in the majority of treated patients (89-93). The success of CAR-T therapy compared with soluble antibody-based approaches are in part due to the fact that CAR-T cells are activated upon target cell encounter, resulting in 10,000-fold proliferation of CAR-T cells in vivo (94) for potent and specific elimination of all target cells. An additional advantage of CAR-T "living therapy" is due to the incorporation of CD28 and/or CD137 costimulatory domains in the CAR design that prevent $\mathrm{T}$ cell exhaustion and promote persistence of memory CAR-T cells that provide continuous surveillance against cancer recurrence $(87,88,95)$. In PV, the anti-DSG3 BCR specifically marks the autoreactive B cell population; thus, the DSG3 CAAR should confer potent and persistent antigen-specific B cell killing in pemphigus, leading to clinical and serologic remission.

To evaluate the feasibility of the CAAR approach (85), truncated fragments of the DSG3 extracellular domain, consisting of EC1-3, EC1-4, and EC1-5, were expressed in primary human $\mathrm{T}$ cells as chimeric receptors fused to CD137/CD3 $\zeta$ signaling domains. All three DSG3 CAARs were shown to be conformational, based on binding of an anti-DSG3 mAb that only recognizes mature conformational DSG3 (96). DSG3 EC1-3 and DSG3 EC1-4 CAAR T cells were shown to specifically kill murine hybridomas and human B cell lines expressing anti-DSG3 BCRs, whereas DSG3 EC1-5 CAAR-T cells did not exhibit significant target cell cytolysis. This lack of target cell cytolysis was thought to be due to the variable surface expression and/or the suboptimal length of the EC1-5 CAAR, which is predicted to create an intermembrane distance greater than that required for optimal $\mathrm{T}$ cell activation (97). In a PV hybridoma mouse model (21), characterized by suprabasal epithelial blistering due to hybridoma secretion of polyclonal antiDSG3 antibodies targeting DSG3 EC1, EC2, and the EC3/4 interface, representing the broad range of B cell epitopes and BCR affinities that could be targeted in human patients, DSG3 CAAR-T cells eliminated all anti-DSG3 $\mathrm{BCR}^{+}$cells, resulting in elimination of serum anti-DSG3 antibody titers and prevention of epithelial blistering. No off-target toxicity was observed against desmosome-expressing tissues or Fc $\gamma$ receptor-expressing cells, which could bind anti-DSG3 antibodies and potentially become targets for CAAR-T cell cytotoxicity. Additionally, CAAR-T cells were effective in eliminating anti-DSG3 B cells both in vitro and in vivo, even in the presence of anti-DSG3 antibodies that could block DSD3 CAAR interactions or lead to DSG3 CAAR-T clearance. The effect of soluble autoantibodies on CAAR-T function is complicated, as different anti-DSG3 mAbs can have differing effects on CAAR-T function, including direct inhibition of cytotoxicity, endocytosis of DSG3 CAAR-Ts (which can be replenished by newly synthesized CAAR-Ts), or stimulation of CAAR-T proliferation and cytokine production. Collectively, given the ability of CAAR-T cells to proliferate and synthesize new CAAR-T molecules, the data suggest that soluble anti-DSG3 antibodies likely would not prevent and in fact may enhance CAAR-T function.

The DSG3 CAAR approach offers several therapeutic advantages and fewer toxicities compared with antibody-based B cell depletion or CAR-T therapy of cancer. First, CAAR T cells can expand and persist indefinitely in vivo to eliminate DSG3-autoreactive B cells, presumably with superior potency and durability compared with antibody-mediated B cell depletion. Second, antigen-specific B cell depletion avoids the risks of generalized immune suppression associated with pan-B cell depletion. Moreover, commonly observed side effects of CAR treatment of cancer, such as cytokine release syndrome $(98,99)$, are not likely to apply in the autoimmune setting, since cytokine release syndrome is associated with high tumor cell burden and the frequency of DSG3-autoreactive B cells is several log-fold lower compared with leukemia cells (100). 
Consistently, tumor lysis syndrome associated with rituximab therapy of cancer has never been reported in pemphigus patients after rituximab treatment given their normal B cell counts prior to therapy, indicating that the target cell frequency may play an important role in the development of cytokine release syndrome and tumor lysis syndrome. Additionally, target cell escape in cancer has been attributed to loss of CD19 expression or mutation of CD19 (101), which is likely irrelevant in PV since B cells that shed their BCRs can no longer receive activation signals through the $\mathrm{BCR}$ to mature into antibody-secreting cells, and $\mathrm{B}$ cells that mutate their BCRs to no longer bind DSG3 by definition become irrelevant to disease. Human clinical trials will be necessary to determine whether CAAR technology can offer safe and long-lasting disease remission.

\section{Future directions}

In summary, as a result of decades of basic, translational, and clinical research, PV has emerged as one the best-characterized human autoimmune diseases. As potent and feasible strategies for antigenspecific targeting progress from preclinical proof of concept to clinical trials, the ideal of safe and effective remissions of autoimmunity by targeting only the disease-causing immune populations is finally coming within our reach. A broad range of approaches to target the pathogenic autoantibodies or the immune cells that stimulate their production are in preclinical and clinical development for PV, offering hope that the "row of tombstones" that describes the histology of PV will eventually have only diagnostic but not prognostic significance.

\section{Acknowledgments}

The authors are grateful for research support from the Penn Institute for Immunology, National Institute of Arthritis and Musculoskeletal and Skin Diseases (NIAMS AR064220 and AR068288), Dermatology Foundation, and Deutsche Forschungsgemeinschaft (EL711/1-1). The contents are solely the responsibility of the authors and do not necessarily represent the official views of NIAMS or the National Institutes of Health.

Address correspondence to: Aimee S. Payne, 421 Curie Boulevard, 1009 Biomedical Research Building, Philadelphia, Pennsylvania 19104, USA. Phone: 215.746.4488; E-mail: aimee.payne@uphs.upenn.edu.

1. National Institutes of Health Report of the Director 2012. National Institute of Health. https://report.nih.gov/pdf/NIH_Biennial_ Report_2012.pdf. Accessed February 10, 2017.

2. Leshem YA, Gdalevich M, Ziv M, David M, Hodak E, Mimouni D. Opportunistic infections in patients with pemphigus. $J$ Am Acad Dermatol. 2014;71(2):284-292.

3. Rosenberg FR, Sanders S, Nelson CT. Pemphigus: a 20-year review of 107 patients treated with corticosteroids. Arch Dermatol. 1976;112(7):962-970.

4. Langan SM, Smeeth L, Hubbard R, Fleming KM, Smith CJ, West J. Bullous pemphigoid and pemphigus vulgaris--incidence and mortality in the UK: population based cohort study. BMJ. 2008;337:a180.

5. Payne AS, Stanley JR. Pemphigus. In: Wolff K, Goldsmith LA, Katz SI, Gilchrest B, Paller AS, Leffell DJ eds. Dermatology in General Medicine. New York: McGraw Hill; 2012: 54.

6. Amagai M, Tsunoda K, Zillikens D, Nagai T, Nishikawa T. The clinical phenotype of pemphigus is defined by the anti-desmoglein autoantibody profile. J Am Acad Dermatol. 1999;40(2 Pt 1):167-170.

7. Amagai M, et al. Usefulness of enzyme-linked immunosorbent assay using recombinant desmogleins 1 and 3 for serodiagnosis of pemphigus. Br J Dermatol. 1999;140(2):351-357.

8. Schmidt E, et al. Novel ELISA systems for antibodies to desmoglein 1 and 3: correlation of disease activity with serum autoantibody levels in individual pemphigus patients. Exp Dermatol. 2010;19(5):458-463.

9. Ahmed AR, et al. Monopathogenic vs multipathogenic explanations of pemphigus pathophysiology. Exp Dermatol. 2016;25(11):839-846.

10. Anhalt GJ, Labib RS, Voorhees JJ, Beals TF, Diaz LA. Induction of pemphigus in neonatal mice by passive transfer of IgG from patients with the disease. N Engl J Med. 1982;306(20):1189-1196.

11. Amagai M, Karpati S, Prussick R, Klaus-Kovtun V, Stanley JR. Autoantibodies against the amino-terminal cadherin-like binding domain of pemphigus vulgaris antigen are pathogenic. J Clin Invest. 1992;90(3):919-926.

12. Amagai M, Hashimoto T, Shimizu N, Nishikawa T. Absorption of pathogenic autoantibodies by the extracellular domain of pemphigus vulgaris antigen (Dsg3) produced by baculovirus. J Clin Invest. 1994;94(1):59-67.

13. Chen Y, Chernyavsky A, Webber RJ, Grando SA, Wang PH. Critical role of the neonatal Fc receptor (FcRn) in the pathogenic action of antimitochondrial autoantibodies synergizing with anti-desmoglein autoantibodies in pemphigus vulgaris. $J$ Biol Chem. 2015;290(39):23826-23837.

14. Mascaró JM, et al. Mechanisms of acantholysis in pemphigus vulgaris: role of IgG valence. Clin Immunol Immunopathol. 1997;85(1):90-96.

15. Payne AS, et al. Genetic and functional characterization of human pemphigus vulgaris monoclonal autoantibodies isolated by phage display. J Clin Invest. 2005;115(4):888-899. 
16. Anhalt GJ, Till GO, Diaz LA, Labib RS, Patel HP, Eaglstein NF. Defining the role of complement in experimental pemphigus vulgaris in mice. J Immunol. 1986;137(9):2835-2840.

17. Funakoshi T, Lunardon L, Ellebrecht CT, Nagler AR, O'Leary CE, Payne AS. Enrichment of total serum IgG4 in patients with pemphigus. Br J Dermatol. 2012;167(6):1245-1253.

18. Futei Y, Amagai M, Ishii K, Kuroda-Kinoshita K, Ohya K, Nishikawa T. Predominant IgG4 subclass in autoantibodies of pemphigus vulgaris and foliaceus. J Dermatol Sci. 2001;26(1):55-61.

19. Aalberse RC, Schuurman J. IgG4 breaking the rules. Immunology. 2002;105(1):9-19.

20. Bruhns $P$, et al. Specificity and affinity of human Fcgamma receptors and their polymorphic variants for human IgG subclasses. Blood. 2009;113(16):3716-3725.

21. Tsunoda $\mathrm{K}$, et al. Induction of pemphigus phenotype by a mouse monoclonal antibody against the amino-terminal adhesive interface of desmoglein 3. J Immunol. 2003;170(4):2170-2178.

22. Heupel WM, Zillikens D, Drenckhahn D, Waschke J. Pemphigus vulgaris IgG directly inhibit desmoglein 3-mediated transinteraction. J Immunol. 2008;181(3):1825-1834.

23. Di Zenzo G, et al. Pemphigus autoantibodies generated through somatic mutations target the desmoglein-3 cis-interface. J Clin Invest. 2012;122(10):3781-3790.

24. Aoyama Y, Kitajima Y. Pemphigus vulgaris-IgG causes a rapid depletion of desmoglein 3 (Dsg3) from the Triton X-100 soluble pools, leading to the formation of Dsg3-depleted desmosomes in a human squamous carcinoma cell line, DJM-1 cells. J Invest Dermatol. 1999;112(1):67-71.

25. Mao X, Choi EJ, Payne AS. Disruption of desmosome assembly by monovalent human pemphigus vulgaris monoclonal antibodies. J Invest Dermatol. 2009;129(4):908-918.

26. Oktarina DA, van der Wier G, Diercks GF, Jonkman MF, Pas HH. IgG-induced clustering of desmogleins 1 and 3 in skin of patients with pemphigus fits with the desmoglein nonassembly depletion hypothesis. Br J Dermatol. 2011;165(3):552-562.

27. Saito M, et al. Signaling dependent and independent mechanisms in pemphigus vulgaris blister formation. PLoS One. 2012;7(12):e50696.

28. Berkowitz P, Hu P, Warren S, Liu Z, Diaz LA, Rubenstein DS. p38MAPK inhibition prevents disease in pemphigus vulgaris mice. Proc Natl Acad Sci USA. 2006;103(34):12855-12860.

29. Mao X, Sano Y, Park JM, Payne AS. p38 MAPK activation is downstream of the loss of intercellular adhesion in pemphigus vulgaris. J Biol Chem. 2011;286(2):1283-1291.

30. Spindler V, et al. Peptide-mediated desmoglein 3 crosslinking prevents pemphigus vulgaris autoantibody-induced skin blistering. J Clin Invest. 2013;123(2):800-811.

31. Mao X, Li H, Sano Y, Gaestel M, Mo Park J, Payne AS. MAPKAP kinase 2 (MK2)-dependent and -independent models of blister formation in pemphigus vulgaris. J Invest Dermatol. 2014;134(1):68-76.

32. Yeh SW, et al. Pathogenic human monoclonal antibody against desmoglein 3. Clin Immunol. 2006;120(1):68-75.

33. Qian Y, Diaz LA, Ye J, Clarke SH. Dissecting the anti-desmoglein autoreactive B cell repertoire in pemphigus vulgaris patients. J Immunol. 2007;178(9):5982-5990.

34. Cho MJ, et al. Shared VH1-46 gene usage by pemphigus vulgaris autoantibodies indicates common humoral immune responses among patients. Nat Commun. 2014;5:4167.

35. Boggon TJ, Murray J, Chappuis-Flament S, Wong E, Gumbiner BM, Shapiro L. C-cadherin ectodomain structure and implications for cell adhesion mechanisms. Science. 2002;296(5571):1308-1313.

36. Harrison OJ, et al. Structural basis of adhesive binding by desmocollins and desmogleins. Proc Natl Acad Sci USA. 2016;113(26):7160-7165.

37. Veldman C, Stauber A, Wassmuth R, Uter W, Schuler G, Hertl M. Dichotomy of autoreactive Th1 and Th2 cell responses to desmoglein 3 in patients with pemphigus vulgaris (PV) and healthy carriers of PV-associated HLA class II alleles. J Immunol. 2003;170(1):635-642.

38. Veldman $\mathrm{CM}$, et al. $\mathrm{T}$ cell recognition of desmoglein 3 peptides in patients with pemphigus vulgaris and healthy individuals J Immunol. 2004;172(6):3883-3892.

39. Sarig O, et al. Population-specific association between a polymorphic variant in ST18, encoding a pro-apoptotic molecule, and pemphigus vulgaris. J Invest Dermatol. 2012;132(7):1798-1805.

40. Ahmed AR, et al. Major histocompatibility complex haplotype studies in Ashkenazi Jewish patients with pemphigus vulgaris. Proc Natl Acad Sci USA. 1990;87(19):7658-7662.

41. Sinha AA, et al. A newly characterized HLA DQ beta allele associated with pemphigus vulgaris. Science. 1988;239(4843):1026-1029.

42. Ahmed AR, et al. Major histocompatibility complex haplotypes and class II genes in non-Jewish patients with pemphigus vulgaris. Proc Natl Acad Sci USA. 1991;88(11):5056-5060.

43. Niizeki H, et al. HLA-DQA1, -DQB1 and -DRB1 genotyping in Japanese pemphigus vulgaris patients by the PCR-RFLP method. Tissue Antigens. 1994;44(4):248-251.

44. Miyagawa S, Higashimine I, Iida T, Yamashina Y, Fukumoto T, Shirai T. HLA-DRB1*04 and DRB1*14 alleles are associated with susceptibility to pemphigus among Japanese. J Invest Dermatol. 1997;109(5):615-618.

45. Wucherpfennig KW, et al. Structural basis for major histocompatibility complex (MHC)-linked susceptibility to autoimmunity: charged residues of a single MHC binding pocket confer selective presentation of self-peptides in pemphigus vulgaris. Proc Natl Acad Sci USA. 1995;92(25):11935-11939.

46. Veldman C, Höhne A, Dieckmann D, Schuler G, Hertl M. Type I regulatory T cells specific for desmoglein 3 are more frequently detected in healthy individuals than in patients with pemphigus vulgaris. J Immunol. 2004;172(10):6468-6475

47. Wada N, et al. Aire-dependent thymic expression of desmoglein 3, the autoantigen in pemphigus vulgaris, and its role in T-cell tolerance. J Invest Dermatol. 2011;131(2):410-417.

48. Carson PJ, Hameed A, Ahmed AR. Influence of treatment on the clinical course of pemphigus vulgaris. J Am Acad Dermatol. 1996;34(4):645-652.

49. Murrell DF, et al. Consensus statement on definitions of disease, end points, and therapeutic response for pemphigus. J Am Acad Dermatol. 2008;58(6):1043-1046. 
50. Martin LK, Werth VP, Villaneuva EV, Murrell DF. A systematic review of randomized controlled trials for pemphigus vulgaris and pemphigus foliaceus. J Am Acad Dermatol. 2011;64(5):903-908.

51. Ahmed AR, Dahl MV. Consensus statement on the use of intravenous immunoglobulin therapy in the treatment of autoimmune mucocutaneous blistering diseases. Arch Dermatol. 2003;139(8):1051-1059.

52. Tan-Lim R, Bystryn JC. Effect of plasmapheresis therapy on circulating levels of pemphigus antibodies. J Am Acad Dermatol. 1990;22(1):35-40

53. Søndergaard K, Carstens J, Jørgensen J, Zachariae H. The steroid-sparing effect of long-term plasmapheresis in pemphigus. Acta Derm Venereol. 1995;75(2):150-152.

54. Turner MS, Sutton D, Sauder DN. The use of plasmapheresis and immunosuppression in the treatment of pemphigus vulgaris. J Am Acad Dermatol. 2000;43(6):1058-1064.

55. Colliou N, et al. Long-term remissions of severe pemphigus after rituximab therapy are associated with prolonged failure of desmoglein B cell response. Sci Transl Med. 2013;5(175):175ra30.

56. Ahmed AR, Spigelman Z, Cavacini LA, Posner MR. Treatment of pemphigus vulgaris with rituximab and intravenous immune globulin. N Engl J Med. 2006;355(17):1772-1779.

57. Mouquet H, et al. B-cell depletion immunotherapy in pemphigus: effects on cellular and humoral immune responses. J Invest Dermatol. 2008;128(12):2859-2869.

58. Eming R, Nagel A, Wolff-Franke S, Podstawa E, Debus D, Hertl M. Rituximab exerts a dual effect in pemphigus vulgaris J Invest Dermatol. 2008;128(12):2850-2858.

59. Lunardon L, et al. Adjuvant rituximab therapy of pemphigus: a single-center experience with 31 patients. Arch Dermatol. 2012;148(9):1031-1036.

60. Feldman RJ, Ahmed AR. Relevance of rituximab therapy in pemphigus vulgaris: analysis of current data and the immunologic basis for its observed responses. Expert Rev Clin Immunol. 2011;7(4):529-541.

61. Tony HP, et al. Safety and clinical outcomes of rituximab therapy in patients with different autoimmune diseases: experience from a national registry (GRAID). Arthritis Res Ther. 2011;13(3):R75.

62. Leandro MJ, Cambridge G, Ehrenstein MR, Edwards JC. Reconstitution of peripheral blood B cells after depletion with rituximab in patients with rheumatoid arthritis. Arthritis Rheum. 2006;54(2):613-620.

63. Ohyama B, et al. Epitope spreading is rarely found in pemphigus vulgaris by large-scale longitudinal study using desmoglein 2-based swapped molecules. J Invest Dermatol. 2012;132(4):1158-1168.

64. Hammers CM, et al. Persistence of anti-desmoglein $3 \operatorname{IgG}(+)$ B-cell clones in pemphigus patients over years. J Invest Dermatol. 2015;135(3):742-749.

65. Heupel WM, Müller T, Efthymiadis A, Schmidt E, Drenckhahn D, Waschke J. Peptides targeting the desmoglein 3 adhesive interface prevent autoantibody-induced acantholysis in pemphigus. J Biol Chem. 2009;284(13):8589-8595.

66. Langenhan J, et al. Specific immunoadsorption of pathogenic autoantibodies in pemphigus requires the entire ectodomains of desmogleins. Exp Dermatol. 2014;23(4):253-259.

67. Amagai M, Ishii K, Hashimoto T, Gamou S, Shimizu N, Nishikawa T. Conformational epitopes of pemphigus antigens (Dsg1 and Dsg3) are calcium dependent and glycosylation independent. J Invest Dermatol. 1995;105(2):243-247.

68. Ishii K, Lin C, Siegel DL, Stanley JR. Isolation of pathogenic monoclonal anti-desmoglein 1 human antibodies by phage display of pemphigus foliaceus autoantibodies. J Invest Dermatol. 2008;128(4):939-948.

69. Zöllner S, Pablik E, Druml W, Derfler K, Rees A, Biesenbach P. Fibrinogen reduction and bleeding complications in plasma exchange, immunoadsorption and a combination of the two. Blood Purif. 2014;38(2):160-166.

70. Miller SD, Turley DM, Podojil JR. Antigen-specific tolerance strategies for the prevention and treatment of autoimmune disease. Nat Rev Immunol. 2007;7(9):665-677.

71. Payne AS, Siegel DL, Stanley JR. Targeting pemphigus autoantibodies through their heavy-chain variable region genes. J Invest Dermatol. 2007;127(7):1681-1691.

72. Nimmerjahn F, Ravetch JV. Fcgamma receptors as regulators of immune responses. Nat Rev Immunol. 2008;8(1):34-47.

73. Lutterotti A, et al. Antigen-specific tolerance by autologous myelin peptide-coupled cells: a phase 1 trial in multiple sclerosis. $S c i$ Transl Med. 2013;5(188):188ra75.

74. Werth VP, et al. An open-label phase I clinical study to assess the safety of PI-0824 in patients with pemphigus vulgaris. J Invest Dermatol. 2005;125(1):A5.

75. Boyle RJ, et al. Venom immunotherapy for preventing allergic reactions to insect stings. Cochrane Database Syst Rev. 2012;10:CD008838

76. Francis JN, et al. Grass pollen immunotherapy: IL-10 induction and suppression of late responses precedes IgG4 inhibitory antibody activity. J Allergy Clin Immunol. 2008;121(5):1120-1125.e2.

77. James LK, et al. Long-term tolerance after allergen immunotherapy is accompanied by selective persistence of blocking antibodies. J Allergy Clin Immunol. 2011;127(2):509-516.e1.

78. MacDonald KG, et al. Alloantigen-specific regulatory T cells generated with a chimeric antigen receptor. J Clin Invest. 2016;126(4):1413-1424.

79. Malhotra S, Kovats S, Zhang W, Coggeshall KM. B cell antigen receptor endocytosis and antigen presentation to T cells require Vav and dynamin. J Biol Chem. 2009;284(36):24088-24097.

80. Proby CM, et al. Development of chimeric molecules for recognition and targeting of antigen-specific B cells in pemphigus vulgaris. Br J Dermatol. 2000;142(2):321-330.

81. Beattie BK, Prentice GA, Merrill AR. Investigation into the catalytic role for the tryptophan residues within domain III of Pseudomonas aeruginosa exotoxin A. Biochemistry. 1996;35(48):15134-15142.

82. Yates SP, Merrill AR. Elucidation of eukaryotic elongation factor-2 contact sites within the catalytic domain of Pseudomonas aeruginosa exotoxin A. Biochem J. 2004;379(Pt 3):563-572.

83. Jackson ME, Simpson JC, Girod A, Pepperkok R, Roberts LM, Lord JM. The KDEL retrieval system is exploited by Pseudomonas exotoxin A, but not by Shiga-like toxin-1, during retrograde transport from the Golgi complex to the endoplasmic reticulum. J Cell Sci. 1999;112 (Pt 4):467-475. 
84. Pastan I, Chaudhary V, FitzGerald DJ. Recombinant toxins as novel therapeutic agents. Annu Rev Biochem. 1992;61:331-354.

85. Ellebrecht CT, et al. Reengineering chimeric antigen receptor T cells for targeted therapy of autoimmune disease. Science. 2016;353(6295):179-184.

86. Gross G, Waks T, Eshhar Z. Expression of immunoglobulin-T-cell receptor chimeric molecules as functional receptors with antibody-type specificity. Proc Natl Acad Sci USA. 1989;86(24):10024-10028.

87. Savoldo B, et al. CD28 costimulation improves expansion and persistence of chimeric antigen receptor-modified T cells in lymphoma patients. J Clin Invest. 2011;121(5):1822-1826.

88. Milone MC, et al. Chimeric receptors containing CD137 signal transduction domains mediate enhanced survival of T cells and increased antileukemic efficacy in vivo. Mol Ther. 2009;17(8):1453-1464.

89. Maude SL, et al. Chimeric antigen receptor T cells for sustained remissions in leukemia. N Engl J Med. 2014;371(16):1507-1517.

90. Davila ML, et al. Efficacy and toxicity management of 19-28z CAR T cell therapy in B cell acute lymphoblastic leukemia. Sci Transl Med. 2014;6(224):224ra25.

91. Lee DW, et al. T cells expressing CD19 chimeric antigen receptors for acute lymphoblastic leukaemia in children and young adults: a phase 1 dose-escalation trial. Lancet. 2015;385(9967):517-528.

92. Kochenderfer JN, et al. Chemotherapy-refractory diffuse large B-cell lymphoma and indolent B-cell malignancies can be effectively treated with autologous T cells expressing an anti-CD19 chimeric antigen receptor. J Clin Oncol. 2015;33(6):540-549.

93. Brentjens RJ, et al. Safety and persistence of adoptively transferred autologous CD19-targeted T cells in patients with relapsed or chemotherapy refractory B-cell leukemias. Blood. 2011;118(18):4817-4828.

94. Porter DL, Levine BL, Kalos M, Bagg A, June CH. Chimeric antigen receptor-modified T cells in chronic lymphoid leukemia N Engl J Med. 2011;365(8):725-733.

95. Long AH, et al. 4-1BB costimulation ameliorates $\mathrm{T}$ cell exhaustion induced by tonic signaling of chimeric antigen receptors. Nat Med. 2015;21(6):581-590.

96. Sharma PM, Choi EJ, Kuroda K, Hachiya T, Ishii K, Payne AS. Pathogenic anti-desmoglein monoclonal antibodies demonstrate variable ELISA activity due to preferential binding of mature versus proprotein isoforms of desmoglein 3. J Invest Dermatol. 2009; 129(9): 2309-2312.

97. Choudhuri K, Wiseman D, Brown MH, Gould K, van der Merwe PA. T-cell receptor triggering is critically dependent on the dimensions of its peptide-MHC ligand. Nature. 2005;436(7050):578-582.

98. Fitzgerald JC, et al. Cytokine release syndrome after chimeric antigen receptor T cell therapy for acute lymphoblastic leukemia. Crit Care Med. 2017;45(2):e124-e131.

99. Teachey DT, et al. Identification of predictive biomarkers for cytokine release syndrome after chimeric antigen receptor T-cell therapy for acute lymphoblastic leukemia. Cancer Discov. 2016;6(6):664-679.

100. Nishifuji K, Amagai M, Kuwana M, Iwasaki T, Nishikawa T. Detection of antigen-specific B cells in patients with pemphigus vulgaris by enzyme-linked immunospot assay: requirement of $\mathrm{T}$ cell collaboration for autoantibody production. $J$ Invest Dermatol. 2000;114(1):88-94

101. Ruella M, Maus MV. Catch me if you can: Leukemia Escape after CD19-Directed T Cell Immunotherapies. Comput Struct Biotechnol J. 2016;14:357-362. 